\title{
Coronary Artery Disease in an Asymptomatic Population Undergoing a Multidetector Computed Tomography (MDCT) Coronary Angiography
}

\author{
Ghassan Zaid ${ }^{1}$, Dana Yehudai $^{1}$, Uri Rosenschein ${ }^{1}$ and Abdel-Rauf Zeina ${ }^{2,3 *}$

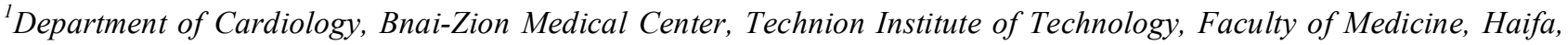 \\ Israel \\ ${ }^{2}$ Department of Radiology and MAR Imaging Institute, Bnai-Zion Medical Center, Technion Institute of Technology, \\ Faculty of Medicine, Haifa, Israel \\ ${ }^{3}$ Department of Radiology, Hillel Yaffe Medical Center, Hadera, Israel
}

\begin{abstract}
Aim: To assess the prevalence of coronary artery disease (CAD) in asymptomatic subjects using multidetector computed tomography (MDCT) and its relationships to demographic and clinical risk factors.

Material and method: We enrolled consecutive asymptomatic volunteers with no evidence of ischemic heart disease that underwent MDCT for the early detection of CAD. All MDCT findings were correlated with demographic and risk factors. A total of 2820 coronary segments were analyzed in 188 asymptomatic subjects (150 males and 38 females), aged $54.4 \pm$ 7.4 years.

Results: A total of $128(68 \%)$ demonstrated MDCT findings compatible with CAD; of these $111(86.7 \%)$ had nonsignificant (diameter stenosis $\leq 50 \%$ ) and $17(13.3 \%$ ) had significant CAD (diameter stenosis $\geq 50 \%$ ). Compared with older subjects (mean age $56 \pm 8$ years), younger subjects had a lower prevalence of MDCT findings of CAD $55.5 \%$ vs. $12.5 \%$, respectively $(P<0.001)$, regardless of risk factors. Males had more CAD (mostly non-significant) compared with females $(109[72.7 \%]$ vs. 19 [50.3\%], respectively; $P=0.007)$. Subjects with $\geq 2$ risk factors had a higher prevalence of $\mathrm{CAD}$ in general and significant $\mathrm{CAD}$ in particular $(P<0.001)$
\end{abstract}

Conclusion: CAD in asymptomatic population seems to be not uncommon. Using MDCT a high prevalence of nonsignificant and low prevalence of significant $\mathrm{CAD}$ was discovered in middle age asymptomatic population.

Keywords: Coronary artery disease, risk factors, multidetector computed tomography.

\section{INTRODUCTION}

Cardiovascular disease is the leading cause of death in the Western population accounting for $38.5 \%$ of all deaths [1]. About $40 \%$ of those who experience an acute coronary syndrome (defined as unstable angina, ST-elevation myocardial infarction or non-ST elevation myocardial infarction) in a given year will die from it. Of notable interest, $50 \%$ of men and $64 \%$ of women who died following acute coronary events had no previous symptoms of this disease [2]. In addition, the estimated cost for treating CHD in the U.S. (direct and indirect) in 2006 will approach $\$ 142.5$ billion [3]. For these reasons, early diagnosis is urgently needed. Until recently, the diagnosis and evaluation of CAD was based on cardiac catheterization performed mostly on ischemic and/or symptomatic patients, both of which are populations with advanced disease. In asymptomatic persons, the emphasis is on the assessment of long-term risk and primary prevention of future clinical disease. Commonly, the first presentation of coronary atherosclerosis is acute myocardial infarction or

*Address correspondence to this author at the Department of Radiology, Hillel Yaffe Medical Center, P.O.B. 169, Hadera 38100, Israel; Tel: + 9724-6304621; Fax: +972-4-6304884: E-mail: raufzeina3@hotmail.com sudden coronary death. Detection of CAD before its 'full blown' manifestation may have a beneficial impact on the natural course of the disease. Hence, clinical risk-factor assessment and stress testing, although they are good prognostic indicators, cannot serve as definitive diagnostic tools. Although risk stratification based on the cardiovascular risk profile is important in identifying cohorts at risk for future cardiovascular events, these data seem to be limited for the diagnosis or exclusion of CAD. The recently developed cardiac CT in 2006 (Electron Beam and Multidetector Computed Tomography [EBCT and MDCT, respectively]), with its high spatial and temporal resolution, is the first accessible and non-invasive imaging technique to enable assessment of CAD in asymptomatic individuals [4-6]. The purpose of this study was to examine the prevalence of atherosclerotic CAD in an asymptomatic population undergoing MDCT and its relationship to demographic and known risk factors.

\section{MATERIALS AND METHODS}

\section{Study Population}

A total of 188 consecutive volunteers, with or without risk factors, underwent MDCT coronary angiography between September 2004 and August 2005 at our institute 
for the early detection of CAD. The study population was comprised of self-referrals or referred by their physician without subjective or objective evidence of ischemic heart disease. Written informed consent was obtained from all subjects included in the study.

Exclusion criteria included a history of previous cardiovascular events (myocardial infarction, unstable angina that required hospitalization, coronary artery bypass grafting, clear evidence of objective ischemia during exercise or imaging testing, invasive coronary angiography, or coronary CT with evidence of CAD), impaired renal function (creatinine level $\geq 1.5 \mathrm{mg} \%$ ), severe lung disease, multiple ectopic beats, atrial fibrillation, heart rate $>75$ beats $/ \mathrm{min}$ despite therapy, and a history of allergic reaction to iodinecontaining contrast. The medical history included cardiac risk factors, previous cardiac investigations and drug treatment. Cardiac risk factors were assessed before the examination and included 1) Diabetes mellitus (defined as a fasting glucose level of $\geq 126 \mathrm{mg} / \mathrm{dl}$ or the need for insulin or oral hypoglycemic agents) [8], 2) Hypertension (defined as blood pressure $\geq 140 / 90 \mathrm{~mm} \mathrm{Hg}$ or the use of anti-hypertension medications) [9], 3) Hyperlipidemia (defined as total cholesterol level $>200 \mathrm{mg} / \mathrm{dl}$ or treatment with lipid-lowering agents) [10], 4) Tobacco use (defined as current smoking), 5) Positive family history (defined as presence of CAD in a first degree relative younger than 55 years for males or less than 65 years for females) [11], and 6) Obesity (defined as body mass index $>30 \mathrm{~kg} / \mathrm{m}$ ) [12]. All patients gave written informed consent to the study protocol after receiving a full explanation of the procedure. Institutional review board approval is not required for retrospective observational studies.

\section{MDCT Scan Protocol}

MDCT was performed using two different MDCT scanners: LightSpeed 16 Pro (112 subjects) and LightSpeed VCT (76 subjects) (GE Healthcare) according to the protocol described earlier $[13,14]$. An appropriate heart rate for the MDCT examination ( $<70$ beats/min) was achieved by oral administration of a beta-blocker (Atenolol 50 to $100 \mathrm{mg}$, based on body mass and basal heart rate) two hours prior to the examination. With the first scanner, the following scanning parameters were applied: detector collimation, $16 \times$ $0.625 \mathrm{~mm} ; 120 \mathrm{kVp} ; 400-500 \mathrm{mAs}$; pitch range, $0.2-0.29$; gantry rotation time, 0.42 second; slice thickness, $0.6 \mathrm{~mm}$. On the second scanner, images were obtained with detector collimation, $64 \times 0.625 \mathrm{~mm} ; 120 \mathrm{kVp} ; 400-500 \mathrm{mAs}$; pitch range, $0.2-0.29$; gantry rotation time, 0.35 second; slice thickness, $0.6 \mathrm{~mm}$. ECG modulation was used in all MDCT examinations (ECG pulsing). The subjects were imaged in the supine position. The distance from the carina to $1 \mathrm{~cm}$ below the diaphragmatic face of the heart was covered. A bolus of 70-90 mL of Iomeron 400 (iomeprol $400 \mathrm{mgI} / \mathrm{mL}$, Bracco) was IV injected $(4 \mathrm{~mL} / \mathrm{s})$ via an 18 -gauge catheter placed in the antecubital vein followed by a bolus of $40 \mathrm{~mL}$ of saline. Scanning delay was determined according to the Smart Prepare Protocol (GE Healthcare) an automatic bolus test; the region of interest was placed on the ascending aorta. The subjects were instructed to maintain an inspiratory breath-hold during which the CT data and ECG trace were acquired.

\section{Image Reconstruction}

Image reconstruction was performed using the retrospective electrocardiographic gating method. Datasets were acquired at phases $45 \%, 75 \%$, and $85 \%$ of the R-R cycle. Other window positions within the cardiac cycle were reconstructed when unsatisfactory results were achieved. The image data sets were processed on a separate workstation (ADW 4.2 and 4.6, General Electric Medical systems, Milwaukee, WI) and analyzed using Curved Multi-Planar Reconstruction (MPR) in multiple planes in addition to the axial source images. Coronary arteries were consensually reviewed by two experienced radiologists and a level 2 certified cardiologist.

\section{DATA ANALYSIS}

CAD was defined as coronary wall atheromatous plaques which could be clearly distinguished from the vessel lumen, with or without luminal reduction, as previously described [15]. Atherosclerotic changes in each coronary segment were classified as 1) calcified (plaques with high density compared to the contrast-enhanced vessel lumen) 2) noncalcified (soft) plaques with lower density, or 3) mixed (calcified and non-calcified morphologies). Luminal narrowing was assessed as non-significant (diameter stenosis $\leq 50 \%$ ), and significant (defined as $>50 \%$ diameter stenosis). For each participant, the number of diseased coronary segments, number of segments with obstructive lesions, and the number of each type of plaque was calculated. Subjects without coronary artery plaques were considered normal.

\section{STATISTICAL ANALYSIS}

All analyses were performed using SPSS 11.5 software. The statistical analyses examined relationships between the different variables (demographic characteristics, risk factors, and MDCT findings) to the presence of significant and nonsignificant CAD. Continuous variables were presented as the mean \pm standard deviation. The $x^{2}$-test was used for categorical variables. Student's $t$-test was used to compare normally distributed groups. The Spearman correlation was used to check for correlations between the ordinal variables and the existence of the disease. The relationship between multiple risk factors and the presence of CAD was assessed with stepwise logistic regression. A two-tailed $P$ value of $<0.05$ was considered significant.

\section{RESULTS}

The study population included $150(79.8 \%)$ males and 38 females with a mean age of $54.4 \pm 7.4$ years. Twenty seven $(14.5 \%)$ subjects had no risk factors, 63 (33.5\%) had 1 risk factor, and 98 (52\%) had $\geq 2$ risk factors for CAD (Table 1). In this population, a positive family history of CAD, hyperlipidemia, and tobacco use were common $(44.6 \%, 43.6 \%$ and $36.7 \%$, respectively). Less common were hypertension (31\%) and diabetes mellitus (12.7\%). The cardiac CT was completed with no reported complications. A total of 2820 coronary segments were analyzed of which $79(2.8 \%)$ were not evaluated due to motion artifacts. The total number of coronary segments with findings of atherosclerotic plaques was 454 (16\%); had soft, calcified, and mixed morphology (Table 2). Of all analyzed subjects, $128(68 \%)$ demonstrated 
Table 1. Baseline Characteristics of the Asymptomatic Study Population

\begin{tabular}{|c|c|}
\hline Variables & Asymptomatic Subjects n=188 (\%) \\
\hline \hline Age (years) ${ }^{1}$ & $54.4 \pm 7.4$ \\
\hline Male & $150(79.8)$ \\
\hline Tobacco use & $69(36.7)$ \\
\hline Hyperlipidemia & $82(43.6)$ \\
\hline Hypertension & $58(30.85)$ \\
\hline Diabetes mellitus & $24(12.7)$ \\
\hline Family history of CAD & $84(44.6)$ \\
\hline No risk factors for CAD & $27(14.5)$ \\
\hline One risk factor for CAD & $63(33.5)$ \\
\hline$\geq 2$ risk factors for CAD & $98(52)$ \\
\hline
\end{tabular}

${ }^{1}$ Values are mean $+\mathrm{SD}, \mathrm{CAD}$; coronary artery disease.

The majority of the study population were middle aged males with $\geq 2$ risk factors for $\mathrm{CAD}$. Common risk factors were positive family history of CAD, hyperlipidemia, and tobacco use.

Table 2. Multidetector Computed Tomography Coronary Angiography Findings

\begin{tabular}{|c|c|}
\hline MDCT Findings & Number (\%) \\
\hline \hline Total evaluated segments & 2820 \\
\hline Non-evaluated segments & $79(2.8)$ \\
\hline Total segments with CAD & $454(16)$ \\
\hline Segments with soft plaques & $123(27.1)$ \\
\hline Segments with calcified plaques & $147(32.4)$ \\
\hline Segments with mixed plaques & $184(40.5)$ \\
\hline Subjects with CAD & $128(68) *$ \\
\hline
\end{tabular}

MDCT; multidetector computed tomography; *percent of the total number of the study cohort $(\mathrm{n}=188)$. Using MDCT, a high prevalence of CAD was detected in our asymptomatic population. The majority of atherosclerotic plaques were mixed and calcified.
MDCT findings compatible with CAD. Of these, 111 $(86.7 \%)$ had non significant and $17(13.3 \%)$ had significant CAD (Fig. 1). Of the 17 patients with significant CAD, 16 (94.1\%) had calcified and mixed morphology plaques. Subjects in different age groups $(40-49,50-59,60-69,70-79$ years) with $\geq 2$ risk factors had a higher prevalence of CAD in general compared with those with only 1 risk factor (from $70 \%$ to $100 \%, P<0.001$ ) (Table 3 and Fig. 2), and significant CAD in particular $(P=0.029)$ (Table $\mathbf{3}$ and Fig. 3). When different age groups were compared, regardless of risk factors, older persons (mean age $56 \pm 8$ years) had a higher prevalence of $\mathrm{CAD}$ of any degree compared with younger persons (mean age $52 \pm 6$ years) $(P=0.007)$ (Table 4), with the exception of those aged 70-79 years with one risk factor who had a prevalence of only $11.1 \%$. After adjustment for other risk factors, males had more CAD (mostly non-significant) compared with females (109 [72.7\%] vs. 19 [50.3\%], respectively; $P=0.007$ ) (Table 4). When we evaluated specific cardiac risk factors, MDCT findings of CAD were observed in about $70 \%-92 \%$ if one of the risk factors was hyperlipidemia $(83 \%)$, hypertension $(74 \%)$, family history of CAD $(75 \%)$, diabetes mellitus $(92 \%)$, or tobacco use $(67 \%)$ (Table 4 and Fig. 4). The presence of CAD of any degree was significantly related to diabetes mellitus $(P=0.007)$ and hyperlipidemia $(P<0.001)$. Significant $C A D$ was significantly related only to a history of diabetes mellitus $(P<0.001)$ and hypertension $(P=0.042)$ (Table 4). On multivariate logistic regression analysis, significant independent predictors of any degree of coronary atherosclerotic disease were male sex (OR $1.46, P=0.001$ ), older age (mean age $\geq 56 \pm 8$ years) (OR 1.09 , $P=0.002$ ), and number of risk factors $(\geq 2)$ (OR 1.84, $P<0.001)$. After adjustment for age and gender, the presence of $\geq 2$ risk factors was still significantly related to the presence of significant CAD.

\section{DISCUSSION}

MDCT angiography is a highly accurate, non-invasive imaging technique with excellent negative predictive value that approaches $100 \%$ which allows CAD to be ruled out $[16,17]$. Hoffmann et al. established the high accuracy of 16-slice MDCT to detect significant obstruction $(>50 \%$ stenosis) with segment-based sensitivity, specificity, and positive and negative predictive values of $95 \%, 98 \%$, $87 \%$, and $99 \%$, respectively, compared with catheter-based

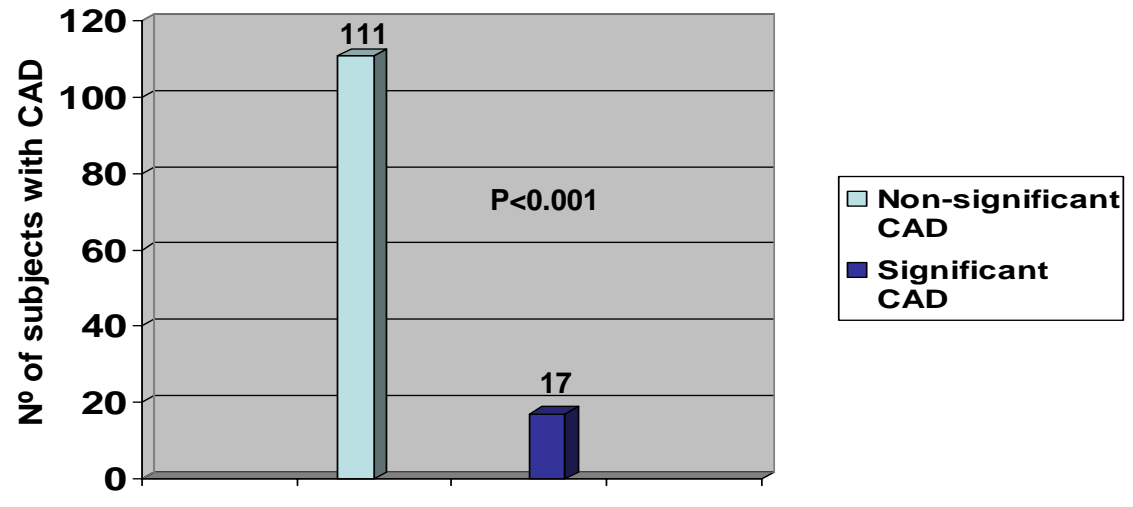

Fig. (1). Severity of coronary artery disease as detected by multidetector computed tomography coronary angiography. A high prevalence of non-significant and low prevalence of significant CAD was observed in our study cohort. 
Table 3. Relationship between the Number of Risk Factors for Coronary Artery Disease and Multidetector Computed Tomography Findings

\begin{tabular}{|c|c|c|c|}
\hline No. of Risk Factors & Normal Coronary Arteries n=60 (\%) & Non-significant CAD n=111 (\%) & Significant CAD n=17 (\%) \\
\hline \hline 0 & $15(55.5)$ & $12(44.5)$ & $0(0)$ \\
\hline 1 & $27(42.8)$ & $30(47.6)$ & $6(9.5)$ \\
\hline 2 & $18(18.4)$ & $69(62.2)$ & $11(64)$ \\
\hline
\end{tabular}

Subjects with $\geq 2$ risk factors had more CAD of any degree than those with fewer risk factors. Even subjects with 0 -1 risk factors had relatively a high prevalence of non- significant CAD. Significant CAD was observed only in subjects with risk factors, and mostly those with $\geq 2$ risk factors.

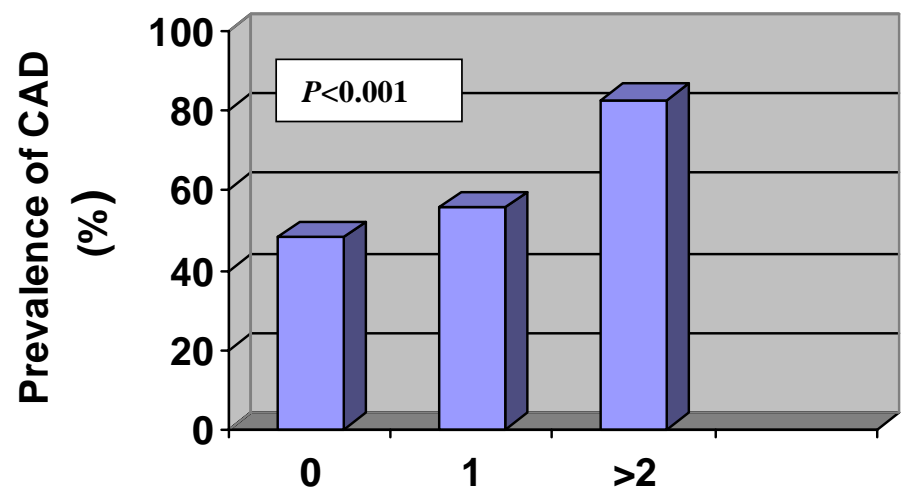

No. of risk factors

Fig. (2). Prevalence of $\mathrm{CAD}$ as it related to the number of risk factors. The presence of $\geq 2$ risk factors was significantly related to high prevalence of CAD ( $P$-value demonstrate the difference between 0 or 1 and $\geq 2$ risk factor). Of note, even subjects with no risk factors had relatively a high proportion of CAD.

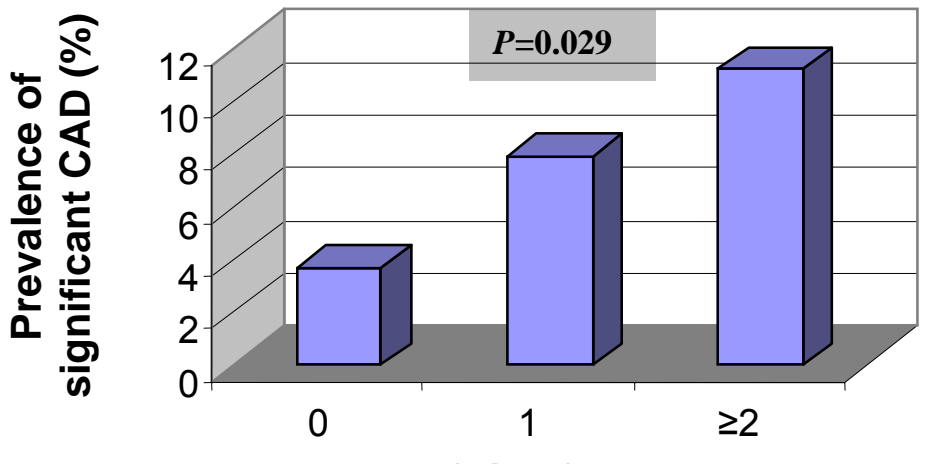

No. of risk factors

Fig. (3). Prevalence of significant $C A D$ as it related to the number of risk factors. A low prevalence of significant CAD was discovered in an asymptomatic middle aged population. However, the presence of $\geq 2$ compared to 0 or 1 risk factors was significantly related to a higher prevalence of significant CAD ( $P$-value demonstrate the difference between 0 or 1 and $\geq 2$ risk factor).

selective coronary angiography [18]. The early discovery of $\mathrm{CAD}$ in the asymptomatic population by this modality may encourage and motivate these patients to be more compliant and to begin or continue to make life style changes. In recent study, multislice coronary CT provided independent prognostic information over base-line clinical risk factors in patients with known and suspected CAD [19-22].

The aim of our study was to discover subclinical CAD in a cohort of asymptomatic individuals using MDCT, and to correlate the findings with demographic characteristics and risk factors. Of interest, coronary atherosclerosis was observed in the majority of our study population $(68 \%)$, and most $(87 \%)$ had non-significant CAD; this finding might explain why they were asymptomatic. Another possible explanation is that the majority of subjects with significant CAD had calcified and mixed morphology plaques which may attribute to the stability and lower vulnerability for active coronary disease and appearance of symptoms. Coronary occlusion and myocardial infarction may, in fact, 
Table 4. Comparison of Demographic and Risk Factors for Coronary Artery Disease by Multidetector Computed Tomography Findings

\begin{tabular}{|c|c|c|c|c|}
\hline Variables & Normal Coronary Arteries $n=60$ & Non-significant CAD n=111 & Significant CAD n=17 & $P$ value \\
\hline Age (years)* & $52.2 \pm 6.3$ & $54.9 \pm 7.8$ & $58.1 \pm 6.3$ & 0.007 \\
\hline Male, n (\%) & $41(27.3)$ & $94(62.7)$ & $15(10)$ & 0.007 \\
\hline Female, n (\%) & $19(50)$ & $17(44.7)$ & $2(5.3)$ & 0.85 \\
\hline Hypertension, n (\%) & $15(25.8)$ & $34(58.6)$ & $9(15.5)$ & 0.020 \\
\hline Family history of CAD n (\%) & $21(25)$ & $57(67.8)$ & $6(7.1 \%)$ & 0.068 \\
\hline Diabetes mellitus, n (\%) & $2(8.3)$ & $14(58.3)$ & $8(33.3)$ & 0.007 \\
\hline Tobacco use, $\mathrm{n}(\%)$ & $23(33.3)$ & $42(60.7)$ & $4(5.8)$ & 0.78 \\
\hline
\end{tabular}

* Values are mean+SD.

MDCT finding of CAD were observed in about 70-92\% of those with the following risk factors: hyperlipdemia ( $83 \%)$, hypertension (74\%), family history of CAD (75\%), diabetes mellitus $(92 \%)$, and tobacco use $(67 \%)$. The presence of CAD of any degree was significantly related to older age (mean $>52 \pm 6$ years), male sex, diabetes mellitus, and hyperlipidemia. Non-significant CAD was significantly related only with diabetes mellitus and a family history of CAD. Significant CAD was significantly related only to a history of diabetes mellitus and hypertension.

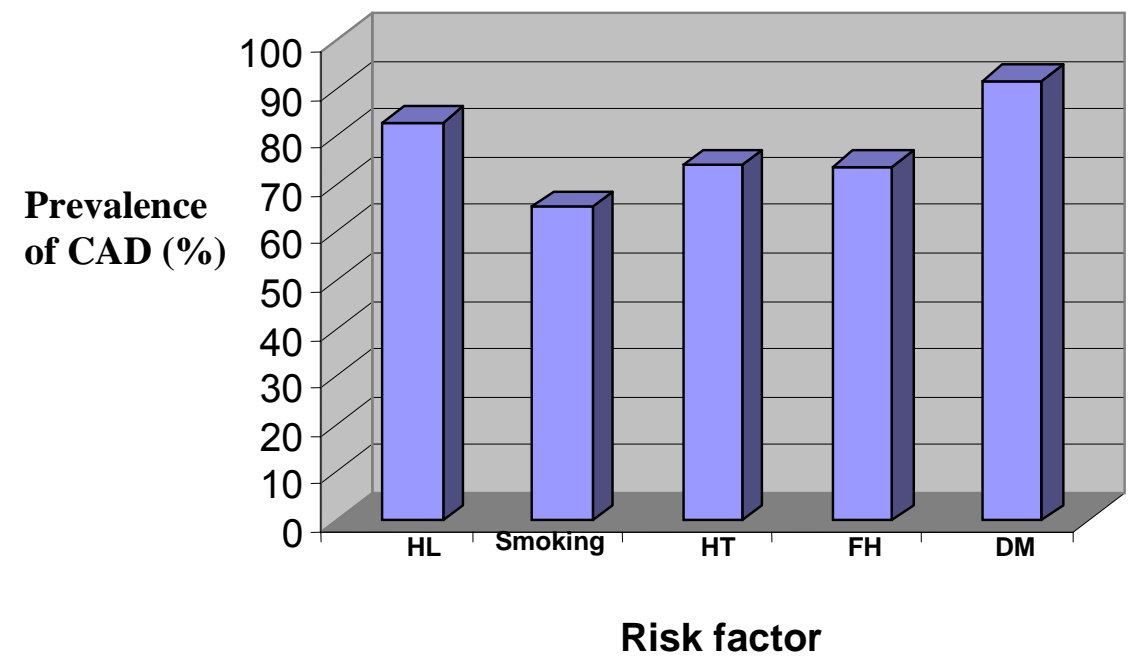

Fig. (4). Prevalence of CAD of any degree as it related to specific risk factors. HL- hyperlipidemia, HT- hypertension, FH- family history of CAD, DM- diabetes mellitus. CAD was observed in $67-92 \%$ of individuals with the risk factors hypertension, family history of CAD, hyperlipidemia, diabetes mellitus, or tobacco use.

arise most frequently from mild to moderate stenosis [22-24]. These angiographic studies showed that $68 \%$ of myocardial infarctions were attributable to so-called "angiographically silent" lesions (luminal narrowing $<50 \%$ ), whereas only $14 \%$ could be assigned to a severe stenosis $(>70 \%)$ [25]. A recent study showed that non-obstructive CAD was indeed an independent predictor of future cardiac events [19]. Thus, our finding of a high prevalence of nonsignificant CAD must be seriously considered.

Our investigation revealed an excellent correlation between the prevalence of CAD and male sex, older age, and number $(\geq 2)$ of risk factors. Although unexpected, this correlation was certainly not observed in age groups older than 70 years. This finding could be explained by the small number [9] of participants in this subgroup. Males had 4-fold more CAD than females of the same age and number of risk factors. This finding might be because women in this age group (mean $54 \pm 7$ years) had a lower probability of the disease compared with men. Nearly one sixth of coronary events in men occur before the age of 55 years. In women who had at least three risk factors, absolute coronary risk exceeded $10 \%$ only after the age of 55 years [26]. In about $13 \%$ of our study population, MDCT demonstrated obstructive coronary atherosclerosis related to older age and a history of two or more risk factors (i.e. diabetes mellitus and hypertension) which mandates further cardiovascular workup (exercise test, isotope scan or coronary angiography). Diabetes mellitus presented in only $12.7 \%$ of our study cohort and was significantly associated with any degree of CAD. Our analysis showed that patients with diabetes had 7fold more CAD than non-diabetics. This finding supports a previous study that showed a high rate of silent ischemia in a retrospective analysis of 1899 asymptomatic patients with type 2 diabetes, aged $\leq 60$ years [27]. Patients with hyperten- 
sion had more significant CAD than normotensive patients. Family history and hyperlipidemia were the dominant risk factors, and were significantly associated only with nonsignificant CAD; this might be the main reason for the high prevalence of non-significant CAD in our study cohort. Other traditional risk factors such as tobacco use were, unexpectedly, not significantly related to any degree of CAD. It could be that, as a single risk factor, tobacco use does not sufficiently affect the disease unless it is combined with other CAD risk factors. Our results correlate well with those of the Framingham Study which indicated that the risk of CAD increases stepwise with the extent of risk-factor clustering [28]. However, data from another studies showed that the presence of risk factors for CAD does not always indicate the presence of the disease [29-31]. Previous study of MDCT in asymptomatic persons [32] suggested that testing could have both desirable effects (changes in behavior) and undesirable ones (worry and increased stress). However, a clinical trial in which coronary CT was used during screening examinations of military personnel found that positive results alone were not a potent motivational force for changes in behavior to reduce coronary risk factors [33]. The primary purpose of screening would be to identify patients whose prognosis could be improved with medical therapy or myocardial revascularization. No randomized controlled studies were found that assessed the value of CT screening in reducing cardiac events. A consensus statement on coronary $\mathrm{CT}$ was issued by American College of Cardiology and the American Heart Association (ACC/AHA) [34]. According to this consensus, coronary CT may be used in "selected" patients with intermediate risk according to the Framingham Risk Score [35]. The Blue Cross, Blue Shield Association Technology Evaluation Center (medical policy on coronary CT) did not recommend the use of coronary CT for screening [36]. None of these guidelines recommend noninvasive testing in an unselected asymptomatic population. In addition, the authors of all these guidelines expressed concern that the results of noninvasive tests could lead to inappropriate or unnecessary diagnostic testing and interventions, including coronary angiography and revascularization, in asymptomatic persons. Hs-CRP is good marker for special group such as high risk and as prognostic marker after cardiac events. It has low sensitivity and specificity for the extent of CAD. For high-risk and low-risk patients, additional noninvasive testing will generally not modify decisions regarding preventive interventions. However, for intermediaterisk patients, those with an absolute 10-year risk of coronary events of approximately 10 to 20 percent according to the Framingham Risk Score [35], noninvasive testing with coronary CT, exercise testing, or perhaps other tests, could improve the assessment of risk and are probably cost-effective. Based on our results and those of other studies, considering the risk of excessive radiation [37] and contrast media, we suggest that coronary CT cannot be used to screen for CAD in the low risk, asymptomatic population.

\section{STUDY LIMITATIONS}

Our study lacked outcome measures and did not establish cost-effective parameters to support or refute the use of MDCT in asymptomatic patients. It also lacked a systematic evaluation of our finding with other imaging modalities for physiological assessments or invasive strategies for lesion confirmation. We did not include the calcium score in the analysis. Our investigation was based on the rationale that calcium scoring sometimes cannot assess the severity of atherosclerosis because soft and mixed plaques, which are not detected by calcium score, might cause significant stenosis of the coronary arteries. The 2007 ACCF/AHA Expert Consensus Document on coronary artery calcium scoring concluded that because of its high false positive rate when applied to low risk populations, it cannot be recommended as a screening tool for the diagnosis of obstructive CHD because of its low specificity [38].

Assessment of the coronary artery lumen on CT is difficult when severely calcified lesions are present. The poor differentiation between contrast-enhanced vessel lumen and high-density calcified plaques may lead to misinterpretation of stenotic lesions and may make some vascular segments unassessable. It is a concern that asymptomatic patient with calcified atherosclerotic changes would be referred for further testing (i.e. stress test) or even undergo coronary intervention, which may be unnecessary.

Patient exposure to ionizing radiation represents, in fact, a major and still debated issue of coronary CT. In patients undergoing coronary $\mathrm{CT}$ dose reducing strategies such as ECG modulation should be used when possible and optimized in accordance with the as low as reasonably achievable principle [39].

\section{CONCLUSION}

CAD in asymptomatic population seems to be not uncommon. Using MDCT a high prevalence of non-significant and low prevalence of significant $\mathrm{CAD}$ was discovered in middle age asymptomatic population. Further investigations are needed to confirm the clinical implications of our findings.

\section{REFERENCES}

[1] Clouse ME, Jersey Chen, Harlan M. Noninvasive screening for coronary artery disease with computed tomography is useful. Circulation 2006; 113: 125-46.

[2] Hurst W. The Heart, arteries and veins. 10th ed. New York, NY: McGraw-Hill 2002.

[3] American heart association statistical update. Heart disease and stroke statistics; 2006 update. Circulation 2006; 113: e85e151.

[4] Achenbach S, Giesler T, Ropert D, et al. Detection of coronary artery stenosis by contrast-enhanced retrospectively electrocardiographically gated, multislice spiral computed tomography. Circulation 2001; 103: 2535-8.

[5] Nieman K, Cademartiri F, Lemos PA, Rolf R, Peter M, Pim J. Reliable noninvasive coronary angiography with fast submillimeter multislice spiral computed tomography. Circulation 2002; 106: $2051-4$.

[6] Knez A, Backer CR, Leber A, et al. Usefulness of multislice spiral computed tomography angiography for determination of coronary artery disease. Am J Cardiol 2001; 88: 1191-4.

[7] O'Malley PG, Taylor AJ, Jackson JL, Doherty TM, Detrano RC. Prognostic value of coronary electron-beam computed tomography for coronary heart disease events in asymptomatic populations. Am J Cardiol 2000; 85: 945-8.

[8] Report of the Expert Committee on the Diagnosis and Classification of Diabetes Mellitus. Diabetes Care 1997; 20: 1183-97.

[9] European Society of Hypertension-European Society of Cardiology guidelines for the management of arterial hypertension. J Hypertens 2003; 21: 1011-3.

[10] Executive Summary of the Third Report of The National Cholesterol Education Program (NCEP) Expert Panel on Detection, 
Evaluation, and Treatment of High Blood Cholesterol in Adults (Adult Treatment Panel III). JAMA 2001; 285: 2486-97.

[11] Taylor AJ, Bindeman J, Feuerstein I, Cao F, Brazaitis M, O’Malley PG. Coronary calcium independently predicts incident premature coronary heart disease over measured cardiovascular risk factors: mean three-year outcomes in the Prospective Army Coronary Calcium (PACC) project. J Am Coll Cardiol 2005; 46: 807-14.

[12] Clinical Guidelines on the Identification, Evaluation, and Treatment of Overweight and Obesity in Adults - the Evidence Report. National Institutes of Health. Obes Res 1998; 6 (Suppl 2): 51S209S.

[13] Schuijf JD, Bax JJ, Salm LP, et al. Noninvasive coronary angiography and assessment of left ventricular function using 16-slice. Am J Cardiol 2005; 95: 571-4.

[14] Escolar E, Weigold G, Fuisz A, Weissman NJ. New imaging techniques for diagnosing coronary artery disease. CMAJ 2006; 14; 174: 487-95

[15] Leber AW, Knez A, Becker A, et al. Accuracy of multidetector spiral computed tomography in identifying and differentiating the composition of coronary atherosclerotic plaques: a comparative study with intracoronary ultrasound. J Am Coll Cardiol 2004; 43: 1241-7.

[16] Schuijf JD, Bax JJ, Shaw LJ, et al. Meta-analysis of comparative diagnostic performance of magnetic resonance imaging and multislice computed tomography for noninvasive coronary angiography. Am Heart J 2006; 151: 404-11.

[17] Mollet NR, Cademartiri F, Mieghem CA, et al. High-resolution spiral computed tomography coronary angiography in patients referred for diagnostic conventional coronary angiography. Circulation 2005; 112: 2318-23.

[18] Hoffmann MH, Shi H, Schmitz BL, Schmid FT, Lieberknecht M, Shulze R. Noninvasive coronary angiography with multislice computed tomography. JAMA 2005; 293: 2471-8.

[19] Pundziute G, Schuijf JD, Jukema JW, et al. Prognostic value of multislice computed tomography coronary angiography in patients with known or suspected coronary artery disease. J Am Coll Cardiol 2007; 49: 62-70.

[20] Min JK, Shaw LJ, Devereux RB, et al. Prognostic value of multidetector coronary computed tomographic angiography for prediction of all cause mortality. J Am Coll Cardiol 2007; 50: 1161-70.

[21] Carrigan TP, Nair D, Schoenhagen P, et al. Prognostic utility of 64-slice computed tomography in patients with suspected but no documented coronary artery disease. Eur Heart J 2009; 30: 36271.

[22] Aldeman EL, Corley SD, Fisher LD, et al. Five-years angiographic follow up of factors associated with progression of coronary artery disease in the Coronary Artery Surgery Study (CASS). CASS Participating Investigations and Staff. J Am Coll Cadiol 1993; 22: 1141-54.

[23] Nobuyoshi M, Tamaka M, Nosaka H, et al. Progression of coronary atherosclerosis: is coronary spasm related to prognosis? $\mathrm{J}$ Am Coll Cardiol 1991; 18: 904-10.

[24] Little WC, Constantinescu M, Applegate RJ, et al. Can coronary angiography predict the site of a subsequent myocardial infarction in patients with mild to moderate coronary artery disease? Circulation 1988; 78: 1157-66.

[25] Falk E, Shah PK, Fuster V. Coronary plaque disruption. Circulation 1995; 92: 657-71.
[26] Vasan RS, Sullivan LM, Wilson PW, et al. Relative importance of borderline and elevated level of coronary heart disease risk factors. Ann Intern Med 2005; 142: 393-402.

[27] Scognamiglio R, Negut C, Ramondo A, Tiengo A, Avogaro A. Detection of coronary artery disease in asymptomatic patients with type 2 diabetes mellitus. J Am Coll Cardiol 2006; 47: 65-71.

[28] Kannel WB. Risk stratification in hypertension: new insights from the Framingham Study. Am J Hypertens 2000; 13: 3S-10S.

[29] Murabito JM, Evans JC, Larson MG, Levy D. Prognosis after the onset of coronary heart disease: an investigation of differences in outcome between the sexes according to initial coronary disease presentation. Circulation 1993; 88: 2548-55.

[30] Michos ED, Vasamreddy CR, Becker CR, et al. Women with a low Framingham risk score and a family history of premature coronary heart disease have a high prevalence of subclinical coronary atherosclerosis. Am Heart J 2005; 150: 1276-81.

[31] Hoffmann U, Moselewski F, Cury RC, et al. Predictive value of 16-slice multidetector spiral computed tomography to detect significant obstructive coronary artery disease in patients at high risk for coronary artery disease: patient-versus segment-based analysis. Circulation 2004; 110: 2638-43.

[32] Wong ND, Detrano RC, Diamond G, et al. Does coronary artery screening by electron beam computed tomography motivate potentially beneficial lifestyle behaviors? Am J Cardiol 1996; 78: 12203.

[33] O'Malley PG, Feuerstein IM, Taylor AJ. Impact of electron beam tomography, with or without case management, on motivation, behavioral change, and cardiovascular risk profile: a randomized controlled trial. JAMA 2003; 289: 2215-23.

[34] Screening for asymptomatic coronary artery disease. In: Preventive Services Task Force. Guide to clinical preventive services. 2nd ed. Baltimore: Williams \& Wilkins 1996; pp. 3-14.

[35] D'Agostino RB Sr, Grundy S, Sullivan LM, Wilson P. Validation of the Framingham coronary heart disease prediction scores: results of a multiple ethnic groups investigation. JAMA 2001; 286: 1807.

[36] O'Rourke RA, Brundage BH, Froelicher VF, et al. American College of Cardiology/American Heart Association Expert Consensus document on electron-beam computed tomography for the diagnosis and prognosis of coronary artery disease. <http://www.ncbi.nlm. nih.gov/pubmed/10880426? itool=EntrezSystem2.PEntrez.Pubmed. Pubmed_ResultsPanel.Pubmed_RVDocSum\&amp;ordinalpos=5> Circulation 2000; 102(1): 126-40.

[37] Einstein AJ, Henzlova MJ, Rajagopalan S. Estimating risk of cancer associated with radiation exposure from 64-Slice computed tomography coronary angiography. JAMA 2007; 298: 317-23.

[38] ACCF/AHA 2007 clinical expert consensus document on coronary artery calcium scoring by computed tomography in global cardiovascular risk assessment and in evaluation of patients with chest pain: a report of the American College of Cardiology Foundation Clinical Expert Consensus Task Force (ACCF/AHA Writing Committee to Update the 2000 Expert Consensus Document on Electron Beam Computed Tomography) developed in collaboration with the Society of Atherosclerosis Imaging and Prevention and the Society of Cardiovascular Computed Tomography. J Am Coll Cardiol 2007; 49: 378-402.

[39] Hausleiter J, Meyer T, Hermann F, et al. Estimated radiation dose associated with cardiac CT angiography. JAMA 2009; 301: 500-7.

Received: December 29, 2009

Revised: January 08, 2010

Accepted: January 12, 2010

(C) Zaid et al.; Licensee Bentham Open.

This is an open access article licensed under the terms of the Creative Commons Attribution Non-Commercial License (http://creativecommons.org/licenses/ by-nc/3.0/) which permits unrestricted, non-commercial use, distribution and reproduction in any medium, provided the work is properly cited. 